\title{
OCULAR SURFACE DISEASE IN SJÖGREN'S SYNDROME: MANAGEMENT IN A SCLERAL LENS CLINICAL PRACTICE
}

Daddi Fadel, DOptom, FSLS ${ }^{1}$ and Melissa Barnett, OD, FAAO, FSLS, FBCLA

${ }^{1}$ Private practice, Rome, Italy.

${ }^{2}$ Department of Ophthalmology and Vision Science, University of California, Davis, Sacramento, CA, USA

Corresponding Author: Daddi Fadel : daddifadel@icloud.com

Submitted: March 14, 2020. Accepted: July 7, 2020. Published: August 25, 2020.

\begin{abstract}
Sjögren's syndrome is a chronic, autoimmune, systemic disease characterized by lymphocytic infiltration and malfunction of the exocrine glands, primarily the lacrimal and salivary glands, resulting in predominant symptoms of dry eye and dry mouth. Sjögren's syndrome is a highly prevalent condition and is one of the most common systemic, rheumatic, autoimmune diseases, affecting up to $1.4 \%$ of adults in the United States, second only to rheumatoid arthritis in its prevalence in North America. Primary Sjögren's syndrome has shown to affect patients' health-related quality-of-life due to dryness, chronic pain, depression, anxiety, physical and mental fatigue, and neuropsychiatric symptoms.

Scleral lenses (SLs) have shown to be significantly beneficial in relieving symptoms and improving quality-of-life in patients with Sjögren's syndrome and dry eye disease. SLs may be used concurrently with the other therapies including ocular lubricants, eyelid hygiene, punctal occlusion, topical prescription medications, and autologous serum.

This manuscript reviews the implication of Sjögren's syndrome on the ocular surface and quality-of-life and describes how SLs, in combination with other treatments, may be beneficial.
\end{abstract}

\section{Key Words: Sjögren Syndrome, Scleral Lens, Dry Eye, Quality-of-Life}

Sjögren's syndrome is a chronic, autoimmune, systemic disease characterized by lymphocytic infiltration and malfunction of the exocrine glands, primarily the lacrimal and salivary glands, resulting in predominant symptoms of dry eye and dry mouth. ${ }^{1}$ Additionally, manifestations of the disease may be found in many systems including musculoskeletal, vascular, pulmonary, gastrointestinal, neurological, lymphatic, gynecological and renal. ${ }^{2}$ The mean age at diagnosis is roughly 50 years. $^{3}$

Sjögren's syndrome is a highly prevalent condition and is one of the most common systemic, rheumatic, autoimmune diseases, affecting up to $1.4 \%$ of US adults, second only to rheumatoid arthritis in its prevalence in North America. ${ }^{4}$ Two forms of the syndrome have been defined: primary Sjögren 
syndrome, in which dysfunction of the exocrine glands occurs in the absence of other autoimmune diseases, whereas secondary Sjögren syndrome is associated with another underlying rheumatic disease, such as systemic lupus erythematosus, rheumatoid arthritis (RA), or scleroderma. ${ }^{3}$

Nine out of 10 patients diagnosed with Sjögren's syndrome are women. ${ }^{5}$ Proposed factors contributing to these sex disparities are differential immune regulation, ${ }^{6-8} \mathrm{X}$-chromosome gene dosage effects, ${ }^{8,9}$ sex hormones, ${ }^{10,11}$ and sex-specific exposure to environmental factors. ${ }^{12,13}$ Additionally, the clinical manifestations of autoimmune diseases can differ between the sexes. ${ }^{5,14}$

The main ocular implication of Sjögren's syndrome is dry eye. ${ }^{15}$ Ocular symptoms include dryness, pain, stinging, burning, itch, epiphora, blurring or interrupted vision eye, irritation (foreign-body sensation) and photophobia. ${ }^{16}$ Other symptoms include chronic pain, depression, anxiety, physical and mental fatigue and neuropsychiatric symptoms. These symptoms affect health-related quality-of-life in patients with Sjögren's syndrome. ${ }^{17,18}$

Various studies demonstrated that SLs are a valuable option in the treatment and therapy of Sjögren's syndrome and dry eye diseases. ${ }^{19,20}$ The benefits of SLs in dry eye disease consistently improved comfort, visual function, and quality-of-life. ${ }^{21}$ Tear Film and Ocular Surface Society DEWS II proposed the use of SLs in the third step of the management algorithm. ${ }^{19}$ This indicates that SLs may be used when other management strategies in the earlier steps fail, or concurrently with the other therapies which include ocular lubricants, eyelid hygiene, punctal occlusion, prescription medications, and autologous serum. ${ }^{19}$

\section{CLASSIFICATION AND MANIFESTATION OF SJÖGREN'S SYNDROME}

Recently, a new classification criterion has been endorsed and published by the American College of Rheumatism and the European League Against Rheumatism. ${ }^{22}$ This criterion has replaced two previous approaches, the American-European Consensus Group, and the American College of Rheumatism classification. ${ }^{22}$ The new criterion integrates elements from the previous practices and eliminates some that are considered invalid. The new classification is based on five objective tests:

- Focal lymphocytic sialadenitis (cut-off/focus score $\geq 1$ );

- Anti-SSA/Ro positivity;

- Ocular staining (cut-off score $\geq 5$, or van Bijsterveld score $\geq 4$, in at least 1 eye);

- Schirmer's test result $\leq 5 \mathrm{~mm} / 5$ minutes in at least 1 eye;

- Unstimulated salivary flow rate $\leq 0.1 \mathrm{ml} /$ minute.

The first two of these are considered the most significant and are given weights of 3 , while the remainder are weighted at 1 each. Patients are classified to have primary Sjögren's syndrome if they have a total score of $\geq 4$, derived from the sum of the weights assigned to each positive test. ${ }^{22}$

Dry eye and xerostomia (dry mouth) are hallmarks of Sjögren's syndrome. Ocular manifestation of Sjögren's syndrome ${ }^{23}$ are summarized in Table 1.

\section{OCULAR SURFACE DISEASE IN SJÖGREN'S SYNDROME}

Ocular surface disease in Sjögren's syndrome is a product of lacrimal functional unit dysfunction. ${ }^{24}$ There are numerous mechanisms for lacrimal gland dysfunction in Sjögren's syndrome. These include cholinergic blockade from autoantibodies to muscarinic acetylcholine receptor 3 , inhibition of acinar secretion by inflammatory cytokines such as IL-1, cytokine-mediated epithelial cell death or replacement of acini by lymphocytes. ${ }^{25}$

Multiple substances are produced by the lacrimal gland that support and protect the ocular surface. ${ }^{26}$ These include growth factors (e.g., EGFs), antimicrobial factors (e.g., lactoferrin, defensins), anti-inflammatory factors (e.g., IL-1RA) and mucins. Reduced concentrations of these substances have been found in tears of patients with Sjögren's syndrome..$^{27,28}$ Inflammatory mediators that cause ocular surface epithelial disease in Sjögren's syndrome include the matrix metalloproteinases (MMPs), inflammatory cytokines and T-helper (Th) cell associated cytokines. Increased production of MMP-3 and MMP-9 by ocular surface cells has been observed in Sjögren's syndrome. ${ }^{27,28}$ 
TABLE 1 Ocular Manifestations of Sjögren's Syndrome

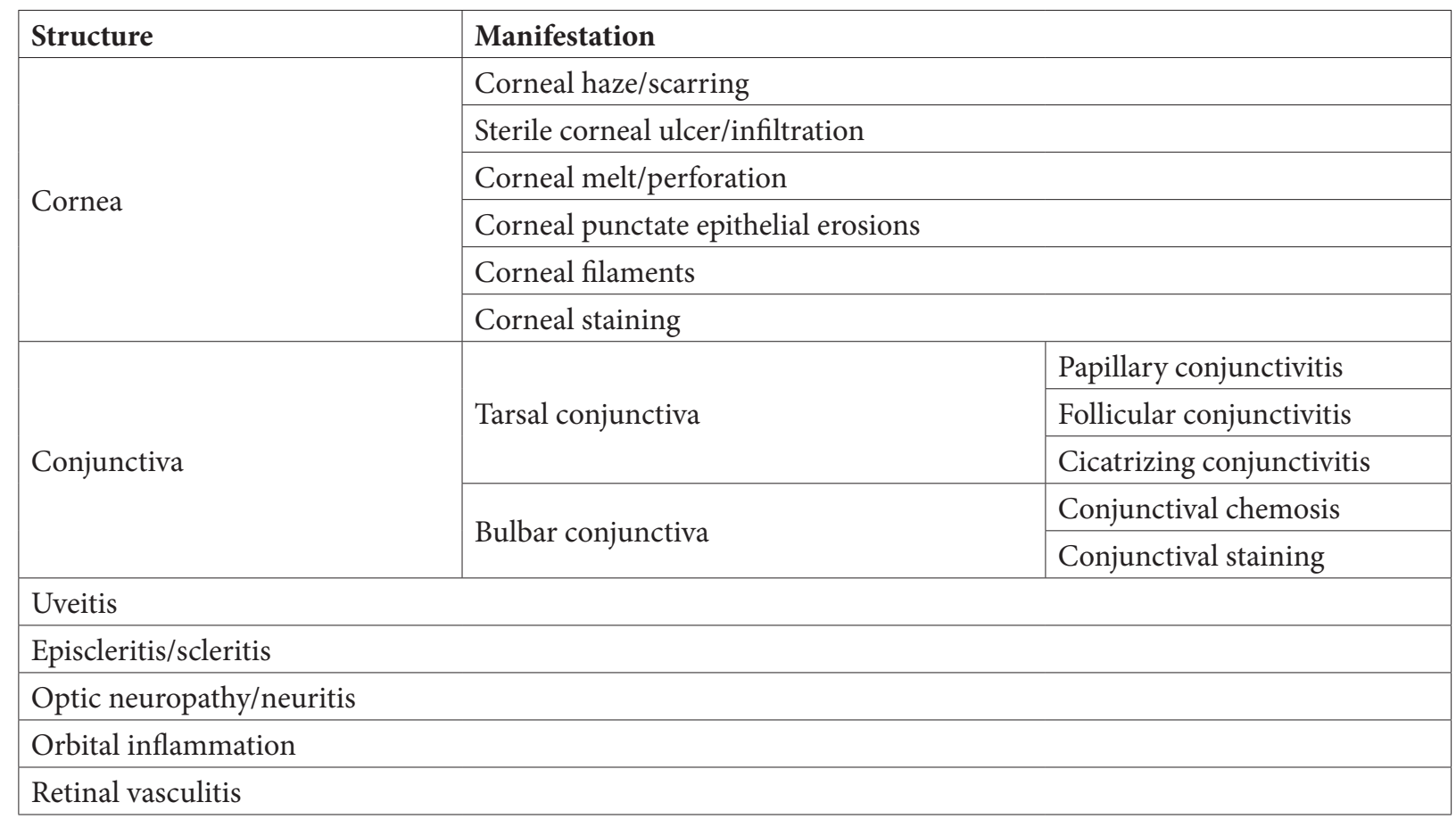

Dry eye is the most common scenario in Sjögren's syndrome. ${ }^{15}$ Henrik Sjögren first described aqueous tear-deficient dry eye in 1933 as an ocular finding in patients with primary Sjögren's syndrome. Ocular symptoms in patients with Sjögren's syndrome include eye irritation (foreign-body sensation) that is constant and can affect their quality-of-life, and photophobia due to tear dysfunction and ocular surface disease. ${ }^{16}$ Other ocular symptoms include dryness, pain, stinging, burning, itch, epiphora, and blurring or interrupted vision.

Ocular testing in Sjögren's syndrome is well defined. The ocular staining score, described by the American College of Rheumatology-European League Against Rheumatism, uses a combination of corneal and conjunctival staining scores with lissamine green and sodium fluorescein and requires a result $\geq 5$ for a positive diagnosis. ${ }^{22}$ An alternative criterion, described by van Bijsterveld, sums corneal and nasal and temporal conjunctival staining scores, each graded from $0-3 .{ }^{29}$ The critical value is $\geq 4$ in at least one eye. Additionally, dry eye disease symptoms must have been present for at least 3 months, as measured on a $0-10$ visual analog scale. ${ }^{22}$
Sjögren's criteria require the American College of Rheumatology-European League Against Rheumatism score ${ }^{22}$ while the Tear Film and Ocular Surface Society DEWS II report does not specify a preferred scale. ${ }^{30}$ Acs et al. suggested that the Tear Film and Ocular Surface Society DEWSII recommended testing (non-invasive TBUT, osmolarity, ocular surface staining, tear meniscus height evaluation and meibomian gland and lipid layer assessment) should be applied to a large group of Sjögren's syndrome patients. ${ }^{31}$

\section{QUALITY-OF-LIFE}

The evaluation of health-related quality-of-life is crucial to understand the burden of the disease and the efficacy of the treatment. Primary Sjögren's syndrome has shown to affect patient's health-related quality-of-life due to dryness, chronic pain, depression, anxiety, physical and mental fatigue and neuropsychiatric symptoms. ${ }^{17,18}$ New findings have emerged from a large, associated-health, quality-of-life study, conducted by Comec et al., in a primary Sjögren's syndrome cohort. ${ }^{17}$ 
The Short Form 36 health survey (SF-36) demonstrated that health-related quality-of-life impairments were severe. Additionally, patients with high systemic activity, assessed with the European League Against Rheumatism Sjögren's Syndrome Disease Activity Index, reported more intense symptoms. Among all symptoms, dryness, pain and fatigue, evaluated with European League Against Rheumatism Sjögren's Syndrome Patient Reported Index, showed the highest scores and the strongest association with healthrelated quality-of-life. Along with these results, the authors concluded that the intensity of symptoms assessed with European League Against Rheumatism Sjögren's Syndrome Patient Reported Index is a stronger determinant of health-related quality-oflife impairment than systemic involvement assessed with the Sjögren's Syndrome Disease Activity Index. Therefore, while future therapeutic trials may use both the Patient Reported and the Disease Activity Indeces, the primary end point criteria should be based on the focus of the population. For example, if the objective is patients' well-being, Patient Reported Index should be used, while the Disease Activity Index, or one of its subscales, would be more appropriate where there is systemic involvement. ${ }^{17}$

Sexual activity may also alter the quality-of-life in women with primary Sjögren's syndrome. ${ }^{32}$ Women with Sjögren's syndrome often experience vaginal dryness and dyspareunia, along with glandular and extraglandular symptoms, ${ }^{33}$ which affect the qualityof-life more than other symptoms. ${ }^{34}$ It has been found that vaginal dryness was ten times greater in Sjögren's syndrome patients reporting an increase in sexual pain and the use of lubricants was five times greater in this group. ${ }^{32}$ Women rarely talk about sexual activity with their physicians, only $17.3 \%$ discussed vaginal problems. ${ }^{32}$ Depending on the cultural environment and social norms, women may be embarrassed to answer questions on sexuality. Depression is the most important predictor of sexual problems. ${ }^{35}$ Referring women to gynecologists and sexologists may be beneficial.

\section{MANAGEMENT WITH SLS}

The 2017 Tear Film and Ocular Surface SocietyDEWS II report suggests SLs as an option for the treatment and therapy of dry eye disease as tertiary management in the staged algorithm. SLs may be used when other management strategies of the earlier steps fail or concurrently with other therapies. ${ }^{19}$

\section{Benefits of SL Wear in Sjögren's Syndrome}

The beneficial response of SL use in dry eye disease has been showed to be consistent regarding comfort, visual function, and quality-of-life. Patients in almost all studies demonstrated an improvement in both the Ocular Surface Disease Index and Visual Function Questionnaire, and significantly improved best-corrected visual acuity. There was no difference in the results based on the origin of dry eye syndrome. The length of SL wearing time was an additional important indicator of the benefits of SLs, with an average of 12 hours per day. ${ }^{21}$ The height of the corneal and limbal vault were found to be a factor contributing to positive outcomes, indicating a corneal vault of 200-300 microns and limbal vault of 100 microns as optimal heights. ${ }^{36}$

A study evaluated the impact of SL wear on dry eye and quality-of-life in 41 eyes majorly with Stevens-Johnson syndrome (53.7\%) and SS (26.8\%). ${ }^{21}$ Diameters of SLs ranged from 16.00 to $17.50 \mathrm{~mm}$, which facilitated successful SL fitting in patients with eyelid scarring (41.7\%). All patients had a significant improvement in best-corrected visual acuity described as a gain of 2 or more Snellen lines. Dry eye symptoms such as irritation, photophobia and foreign-body sensation, assessed by Ocular Surface Disease Index questionnaires were reduced. Additionally, the comfort and quality of best-corrected visual acuity improved. The mean amount of time of SL wear was about 12 hours per day. Additionally, a statistically significant decrease in tear osmolarity was observed between baseline values and after 6 months of SL wear. The reduction in tissue damage, assessed by Bijsterveld corneal staining, was significant after 12 months. Quality-of-life involving physical problems, bodily pain, emotional problems, mental health, energy and vitality, and the general perception of health, measured with the SF-36v2 questionnaire, was also significantly enhanced after 12 months of SL wear. ${ }^{21}$

$\mathrm{Ng}$ and Sorbara presented a case report of a 55-year-old Caucasian male where SLs were used to manage severe ocular dryness secondary to an immune disorder suspected to be Sjögren's syndrome. ${ }^{37}$

J Cont Lens Res Sci Vol Vol 4(2):e12-e22; August 25, 2020

This article is distributed under the terms of the Creative Commons Attribution-Non Commercial 4.0 International License.CFadel and Barnett. 
The patient presented with filamentous keratitis and a persistent epithelial defect with severe chronic superficial punctate keratopathy in both eyes, secondary to his autoimmune condition. The patient was primarily treated with OcunoxTM (Candorvision), HyloTM Gel (Candorvision), Restasis ${ }^{\circledR}$ (cyclosporine ophthalmic emulsion) $0.05 \%$ and autologous serum tears $20 \%$ to manage the ocular dryness. Additionally, the patient had a tarsorrhaphy of the left eyelid to protect corneal integrity. Despite the use of ocular drops and the tarsorrhaphy, dry eye symptoms and signs persisted. After SL wear, the patient reported immediate relief of dry eye symptoms with an improvement of visual acuity. Also, SLs promoted healing and prevented reoccurrence of a persistent epithelial defect. ${ }^{37}$

\section{The Impact of SLs on the Ocular Surface in Sjögren's Syndrome}

Another report assessed basal tear production, corneal sensation, corneal nerve density, and corneal nerve morphology after long-term wear of SLs in two groups, with distorted corneas (DC) and ocular surface disease (OSD) including primary dry eye syndrome, Sjögren's syndrome, ocular graft-versus-host-disease, post-refractive surgery dry eye syndrome, dry eye syndrome associated with blepharitis, and exposure keratopathy. ${ }^{38}$ Significant changes in the lacrimal functional unit were found only in patients with DC probably because of the difference in disease pathophysiology between corneal irregularity and OSD. Furthermore, corneal sensation was increased only in the DC group. It should be stated that corneal sensation varies in dry-eye disease (DED) depending on the etiology. Primary DED is associated with hypoesthesia, ${ }^{39-41}$ while Sjögren's syndrome is associated with hyperesthesia. ${ }^{42,43}$ Corneal nerve density and morphology showed no variation in both groups. ${ }^{38}$

\section{Specific Considerations when Fitting SLs for Sjögren's Syndrome}

Caution is advised when fitting SLs in patients with Sjögren's syndrome. These patients appear more likely to present a higher risk of developing microbial keratitis. ${ }^{44}$ Additionally, patients taking oral and/ or topical corticosteroids which are responsible for reducing immunity have potential risk factors for developing an infection. ${ }^{45-49}$
However, few cases of microbial keratitis due to poor compliance have been reported during scleral lens wear. Compliance with hygiene appears to be higher in SL wearers since their ocular disease necessitates specific ocular hygiene. Therefore, the incidence of microbial keratitis in SL wearers may be lower than contact lens wearers of other modalities. ${ }^{50}$

One case report described the rare occurrence of microsporidial stromal keratitis with polymicrobial keratitis in a patient with secondary Sjögren's syndrome and ocular cicatricial pemphigoid with SL use. ${ }^{51}$ The predisposing factors included dry eye disease, local and systemic immunosuppression, and the use of SLs. The patient had concomitant blepharitis which may have contributed to microbial keratitis. ${ }^{51}$ Drugs used to treat microsporidial keratitis and topical Fumagillin are considered the most effective treatment. ${ }^{52}$ The patient in the case report was treated with topical PHMB 0.02\% (LVPEI Pharmacy, AP, India) and oral albendazole (Taj Pharmaceuticals Ltd, Gujarat, India) because Fumagillin was not available. The therapy failed and patient's condition worsened. Therapeutic penetrating keratoplasty and tarsorrhaphy were performed using an 80-year-old donor corneal tissue with incalculable endothelial cell density. The postoperative graft failed after 4 months and the patient had to wait for a planned keratoprosthesis.

Careful documentation, photodocumentation, and baseline measurements are essential to monitor ocular surface conditions and their potential alteration. If there is concern, a primary evaluation after 4-6 hours of SL wear is recommended to assess the presence of adverse events. ${ }^{49}$ Preservative-free solutions are suggested to avoid preservative sensitivities. SLs should be optimally aligned circumferentially to avoid the influx of debris and air bubbles into the tear reservoir. SLs with a toric back surface, more than four meridians, quadrant-specific design, or molded/ impression design allow an optimal lens alignment to the underlying sclera. Decreasing limbal clearance is also beneficial since this decreases debris influx beneath the lens.

Initially, limiting SL wearing time may be recommended. ${ }^{50}$ If complications do not occur, wearing time may be increased gradually. Practitioners must discuss and clarify with patients the importance of hygiene,

J Cont Lens Res Sci Vol Vol 4(2):e12-e22; August 25, 2020

This article is distributed under the terms of the Creative Commons Attribution-Non Commercial 4.0 International License.(Fadel and Barnett. 
proper care and management of SLs and cleaning and replacing storage cases and other ancillary devices. Recommendations must be confirmed, verified and reinforced during follow-up visits. It is important to recognize that patients may report a higher level of compliance than is factual, revealed by clinical signs and symptoms during examination. Improved collaboration and compliance will increase success rates and minimize complications and infections associated with SLs.

\section{ADDITIONAL TREATMENTS}

SLs are included as the third step in the management algorithm based on a sequence of four steps for the treatment of DED. The more severe the condition, the more likely it is to jump to the next level, with possibility to retain current therapies. ${ }^{19}$ Therefore, SLs may be prescribed when other management strategies of the earlier steps fail or concurrently with other therapies. ${ }^{19}$ Other treatments include ocular lubricants, eyelid hygiene, punctal occlusion, medications, and autologous serum.

\section{Ocular Lubricants}

Ocular lubricants are first line therapy in Sjögren's syndrome ${ }^{54}$ It has been suggested to start with lubricant drops, and if necessary, to select more viscous drops. ${ }^{3}$ The viscosity is obtained from the addition of various enhancing agents (e.g., sodium CMC, HPMC, PVP-K30, PVA, PEG-400, propylene glycol, HPguar, sodium hyoluronate) that provide an increase in lubrication, and an extended residence time on the ocular surface. ${ }^{19}$ In addition to viscous agents, certain tear supplements contain lipids to mimic the lipid component of the tear film. Drops containing lipids may also be beneficial, since the lipid layer prevents tear evaporation. There are a variety of lipids that best mimic natural meibum and include saturated and unsaturated fatty acids, phospholipids, and triglycerides. ${ }^{55}$ The use of biological tear substitutes, especially autologous serum, has been described to be effective in patients with Sjögren's syndrome. ${ }^{19}$

Other options include ophthalmic ointments and hydroxypropyl cellulose inserts. Ointment treatments are the thickest of lubricants and adhere to the ocular surface longer than other tear or gel supplements. They are generally used overnight to provide constant relief of dry eye symptoms during sleep. Hydroxypropyl cellulose is inserted in the inferior cul-de-sac and dissolves with the natural body temperature. Once dissolved, it creates a thicker tear film. It may be applied in a one-daily dose in the morning, however, when used overnight it may perform better. The thickness of these two treatments may induce blurred vision. ${ }^{54}$

Recent reports have shown the effectiveness of tear supplementation in dry eye patients. ${ }^{56,57}$ A study compared the effect of three artificial tear substitutes on the signs, symptoms and inflammatory status in patients with dry eye disease and found that all therapies reduced signs and ameliorated tear stability. However, the treatment with carboxymethylcellulose, glycerine, castor oil, L-carnitine and erythritol demonstrated the greatest tendency to reduce inflammatory biomarker levels. $^{57}$

Topical lubricants are frequently used in patients with Sjögren's syndrome; $85 \%$ of patients with Sjögren's syndrome in North America use topical lubricants. Of these patients, $56 \%$ used preserved solutions and $42.3 \%$ used preservative-free solutions. ${ }^{31,58}$ The Tear Film and Ocular Surface Society DEWS II, in the second step of management and treatment recommendations for dry eye, suggests the use of non-preserved ocular lubricants to minimize preservative induced toxicity. ${ }^{19}$ Patients with dry eye may be more vulnerable to preservative toxicity due to a reduced tear volume. ${ }^{54}$

\section{Eyelid Hygiene}

Few studies are available on the effectiveness of eyelid hygiene in the treatment of dry eye disease. However, eyelid hygiene is generally recommended in dry eye disease associated with blepharitis to reduce the bacterial load on the eyelid margin. ${ }^{59}$ It has been noted that about $20 \%$ of patients with Sjögren's syndrome have anterior blepharitis and $52 \%$ have bilateral meibomian gland disfunction. ${ }^{58}$ The bacterial components may include Staphylococcus aureus, Staphylococcus epidermidis, Propionibacterium acnes, Corynebacterium sp., and Moraxella ${ }^{60}$ Hypochlorous acid hygiene solution $(0.01 \%)$ formed by polymorphonuclear neutrophils to kill microorganisms demonstrated to be effective against staphylococcus in biofilm without disrupting 
biofilm structures. ${ }^{61}$ A recent study has confirmed that tea tree oil is effective against Staphylococcus aureus and the eradication of demodex. ${ }^{60}$ Patients who used tea tree oil experienced improvements in Ocular Surface Disease Index and Tear Break Up Time. However, the use of tea tree oil and ivermectin caused irritation and may not be appropriate for all patients. $^{62}$

Even though eyelid hygiene therapy is commonly prescribed by practitioners, patient's compliance seems to be poor. A recent cross-sectional study involving patients with dry eye symptoms, detected that patients were moderately compliant; an improvement in symptoms were noted in patients who used eyelid hygiene. ${ }^{63}$

\section{Punctal Occlusion}

Punctal occlusion is an obstruction of the tear drainage at the level of the canaliculus allowing tears, or tear substitutes, preservation on the ocular surface. ${ }^{54}$ There are no large-scale studies demonstrating the efficacy of plug occlusion. Despite the theoretical blockage of tear drainage that may lead to an extended time of proinflammatory cytokines on the ocular surface, several reports strongly suggest the positive impact of punctal occlusion on dry eye signs and symptoms. ${ }^{19,54}$

A study investigated the differences between two treatments, tear substitutes compared to punctal plugs over 3 months in 42 patients with primary Sjögren's syndrome and showed that in both groups there was a statistically significant improvement in Ocular Surface Disease Index, corneal fluorescein staining, Schirmer's, and Tear Break Up Time scores compared to baseline scores. ${ }^{64}$ However, treatment of inflammation prior to punctal occlusion is essential. ${ }^{19}$

\section{Prescription Medications}

\section{Topical Corticosteroids}

Topical steroids have been shown to be beneficial in dry eye disease related to Sjögren's syndrome management. ${ }^{65,66} \mathrm{~A}$ study of 21 patients with primary and secondary Sjögren's syndrome treated with a nonpreserved topical corticosteroid solution demonstrated a rapid and significant improvement in ocular irritation symptoms. There was also a decrease in corneal fluorescein scores and filamentary keratitis in all patients with this condition. However, after more than 3 months of therapy, increased intraocular pressure and posterior subcapsular cataracts were evident. ${ }^{65}$ Hong et al. treated 53 patients with Sjögren's syndrome with non-preserved 1\% methylprednisolone solution for two weeks, then tapered off the therapy every two weeks until discontinuation. The authors found a significant decrease in fluorescein staining and improvement in Tear Break Up Time and Schirmer testing. They also observed an increase in periodic acid-Schiff-positive cells indicative of mucin secretory goblet cells. No complications were noted during the long-term treatment. ${ }^{66}$

\section{Topical Cyclosporine}

Cyclosporine, an immunomodulatory drug that inhibits the release of proinflammatory cytokines, has shown to be effective in patients with Sjögren's syndrome. ${ }^{67-70}$ In a double-blind randomized control trial, 15 patients with secondary Sjögren's syndrome were treated with topical cyclosporine A $2 \%$ in olive oil and the other 15 patients received a placebo for two months. There was a significant increase in Tear Break Up Time and a reduction in rose bengal staining scores between the two groups. ${ }^{68}$ A multicenter double-blind randomized control trial enrolled 877 patients with Sjögren's syndrome who instilled cyclosporine A at concentrations of $0.05 \%$ or $0.1 \%$ for six months. With either concentration of cyclosporin A, significant amelioration in corneal staining and Schirmer's testing was shown between the two groups. The improved corneal condition led to a significant improvement in the subjective measurements of blurred vision. ${ }^{69}$ Another multicenter double-blind randomized control trial investigated 162 patients with and without Sjögren's syndrome using topical cyclosporine A at $0.05 \%, 0.1 \%, 0.2 \%$, or $0.4 \%$ for 12 weeks and found a significantly improvement in rose bengal staining, superficial punctate keratitis, sandy or gritty sensation, dryness, itching and a decrease of Ocular Surface Disease Index scores. Cyclosporine A $0.1 \%$ presented the most consistent improvement in objective and subjective end points; cyclosporine A $0.05 \%$ gave the most consistent improvement in patient symptoms. ${ }^{70}$ 


\section{Lifitegrast}

Lifitegrast is a small molecule lymphocyte functionassociated antigen- 1 antagonist that inhibits a specific T-cell inflammatory pathway involved in the pathogenesis of dry eye disease. ${ }^{71}$ Lymphocytic infiltrates of $\mathrm{CD}^{+} \mathrm{T}$ cells and $\mathrm{CD} 4^{+} \mathrm{T}$-helper cells have been observed in conjunctival and lacrimal tissue in patients with and without Sjögren's syndrome. ${ }^{72}$

Lifitegrast ophthalmic solution $5.0 \%$ has shown to be effective and safe in dry eye disease. ${ }^{73,74}$ Two studies have been conducted on patients with and without Sjögren's syndrome treated with Lifitegrast $5.0 \%$ or placebo. ${ }^{73,74}$ The first study of 718 subjects treated over 84 days reported a significant improvement from baseline in eye dryness scores and discomfort, but no significant improvement in inferior corneal staining. ${ }^{73}$ A more recent study on 711 participants for 30 days showed that Lifitegrast-treated participants experienced significantly greater improvement from baseline in dry eye scores, itching, foreign-body sensation, and discomfort compared to those receiving a placebo treatment. ${ }^{74}$

\section{Autologous Serum}

The use of autologous serum is indicated in severe dry eye disease, when previous treatments have not succeeded. ${ }^{19}$ Autologous serum contains fibronectin, vitamin A, cytokines, growth factors and antiinflammatory substances, such as interleukin receptor antagonists and inhibitors of MMPs. Autologous serum has been described to be effective in patients with Sjögren's syndrome. ${ }^{75,76}$ Tsubota et al. showed a significant decrease in symptoms, fluorescein and rose bengal staining in Sjögren's syndrome after 4 weeks of $20 \%$ serum eye drops $6-10$ times daily ${ }^{75}$ A study compared clinical efficacies of autologous serum in primary and secondary Sjögren's syndrome. After 4 weeks of treatment, patients with primary Sjögren's syndrome showed significant improvement in symptoms, ocular surface staining scores, and Tear Break Up Time while patients with secondary Sjögren's syndrome demonstrated no improvement. ${ }^{76}$

\section{CONCLUSIONS AND NEW FRONTIERS}

The advent of SLs has extended their prescription and increased the number of conditions that may be considered indications for their use. Their benefits have been widely documented, and related to dry eye disease they appeared consistent vis-à-vis comfort, visual function, and quality-of-life. Prescribing SLs when other treatments fail or in concurrence with other therapies may solve dramatic scenarios such as depression, anxiety, physical and mental fatigue and neuropsychiatric symptoms in patients with Sjögren's syndrome.

Although there have been numerous advances in our knowledge of Sjögren's syndrome, there are many potential areas of investigation. A variety of genetic and environmental risk factors as well as cellular and molecular pathways have been identified. This new knowledge may provide multiple targets for new systemic and ocular therapies. Additionally, technological advances have led to innovations in genetics, genomics and epigenetic research. An example is the characterization and analysis of DNA and RNA in patient samples on a genome-wide scale. These new techniques will help to identify additional risk factors for the diagnosis, treatment and management of Sjögren's syndrome.

\section{GRANT SUPPORT}

This work was self-funded.

\section{DISCLOSURES}

There are no disclosures for both authors.

\section{CONFLICTS OF INTEREST}

There are no conflicts of interest for both authors.

\section{PRIOR PRESENTATION}

This work has not been presented.

\section{REFERENCES}

1. Bloch K, Buchanan W, Wohl M, et al. Sjögren's syndrome: A clinical, pathological and serological study of 62 cases. Medicine (Baltimore) 1965;44:187-31.

2. Kassan S, Moutsopoulos H. Clinical manifestations and early diagnosis of Sjögren's syndrome, Arch Intern Med 2004;164:1275-84.

3. Dumusc A, Rao V, Bowman SJ. Sjögren's syndrome. Medicine 2018;46:126-30. 
4. Helmick C, Felson D, Lawrence R, et al. Estimates of the prevalence of arthritis and other rheumatic conditions in the United States. Arthritis Rheum 2008;58:15-25.

5. Qin B, Wang J, Yang Z, et al. Epidemiology of primary Sjögren's syndrome: a systematic review and metaanalysis. Ann Rheum Dis 2015;74:1983-9.

6. Absher DM, Li X, Waite LL, et al. Genome-wide dna methylation analysis of systemic lupus erythematosus reveals persistent hypomethylation of interferon genes and compositional changes to CD4+ T-cell populations. PLoS Genet 2013;9:e1003678.

7. Greer JM, McCombe PA. The role of epigenetic mechanisms and processes in autoimmune disorders. Biologics 2012;6:307-27.

8. Inoshita M, Numata S, Tajima A, et al. Sex Differences of leukocytes DNA methylation adjusted for estimated cellular proportions. Biol Sex Differ 2015;6:11.

9. Fish EN. The X-files in Immunity: Sex-based differences predispose immune responses. Nat Rev Immunol 2008;8:737-44.

10. Kovats S. Estrogen receptors regulate innate immune cells and signaling pathways. Cell Immunol 2015;294:63-9.

11. McMurray RW, May W. Sex Hormones and systemic lupus erythematosus: review and meta-analysis. Arthritis Rheum 2003;48:2100-10.

12. Porola P, Laine M, Virkki L, et al. The influence of sex steroids on Sjögren's syndrome. Ann N Y Acad Sci 2007;1108:426-32.

13. Selmi C, Lu Q, Humble MC. Heritability versus the role of the environment in autoimmunity. J Autoimmun 2012;39:249-52.

14. Ramírez Sepúlveda JI, Kvarnström M, Brauner S, et al. Difference in clinical presentation between women and men in incident primary Sjögren's syndrome. Biol Sex Diff 2017;8:16.

15. Vivino F. Sjögren's syndrome : Clinical aspects. Clinical Immunology 2017;182:48-54.

16. Uchino, M, Schaumberg, DA. Dry eye disease: impact on quality-of-life and vision. Curr Ophthalmol Rep 2013;1(2):51-57.

17. Cornec D, Devauchelle-Pensec V, Mariette X, et al. Severe health-related quality-of-life impairment inactive primary Sjögren's syndrome is driven by patient-reported outcomes: data from a large therapeutic trial. Arthritis Care Res (Hoboken) 2017;69(4):528-35.

18. Lackner A, Stradner MH, Hermann J, et al. Assessing health-related quality-of-life in primary Sjögren's syndrome-the PSS-QoL. Semin Arthritis Rheum 2018;48(1):105-10.

19. Jones L, Downie LE, Korb D, et al. TFOS DEWS II Management and Therapy Report. Ocul Surf 2017;15(3):575-28.

20. Fadel D, Kramer E. Potential contraindications to scleral lens wear. Cont Lens Anterior Eye 2019;41(1):92-103.

21. La Porta Weber S, Becco de Souza R, Gomes JÁP, Hofling-Lima AL. The use of the Esclera scleral contact lens in the treatment of moderate to severe dry eye disease. Am J Ophthalmol 2016 Mar;163:167-73.

22. Shiboski CH, Shiboski SC, Seror R, et al. 2016 American College of Rheumatology/European League Against Rheumatism Classification Criteria for Primary Sjögren's Syndrome: a consensus and data-driven methodology involving three international patient cohorts. Ann Rheum Dis 2017;76:9-16.

23. Mathews PM, Hahn S, Hessen M, Kim J, et al. Ocular complications of Primary Sjögren syndrome in men. Am J Ophthalmol 2015;160(3):447-52.

24. Pflugfelder, S. What causes dryness in Sjögren's syndrome patients and how can it be targeted? Exp Rev Clin Immunol 2014:10:4, 425-27.

25. Nguyen CQ, Peck AB. Unraveling the pathophysiology of Sjögren syndrome-associated dry eye disease. Ocul Surf 2009;7(1):11-27.

26. Geerling G, MacLennan S, Hartwing D. Autologus serum eye drops for ocular surface disorders. Br J Ophtalmol 2004;88(11):1467-74.

27. Sobrin L, Selzer MG, Lokeshwar BL, et al. Stromelysin (MMP-3) Activates Pro-MMP-9 secreted by corneal epithelial cells. Invest Ophthalmol Vis Sci 2000;41(7):1703-9.

28. Chotikavanich S, de Paiva CS, Li DQ, et al. Production and activity of matrix metalloproteinase-9 on the ocular surface increase in dysfunctional tear syndrome. Invest Ophthalmol Vis Sci 2009;50(7):3203-9.

29. van Bijsterveld O. Diagnostic Tests in the Sicca Syndrome. Arch Ophthalmol 1969;82:10-14.

30. Wolffsohn J, Arita R, Chalmers R, et al. TFOS DEWS II Tear Film Report. Ocul Surf 2017;15:539-74.

31. Acs M, Caffery B, Barnett M, et al. Customary practices in the monitoring of dry eye disease in Sjögren's syndrome. J Optom 2018;11(4):232-41.

32. Isik H, Isik M, Aynioglu O, et al. Are the women with Sjögren's syndrome satisfied with their sexual activity? Rev Bras Reumatol Engl Ed 2017;57(3):210-16. 
33. Mulherin DM, Sheeran TP, Kumararatne DS, et al. Sjögren's syndrome in women presenting with chronic dyspareunia. Br J Obstet Gynaecol1997;104(9):1019-23.

34. Belenguer R, Ramos-Casals M, Brito-Zerón P, et al. Influence of clinical and immunological parameters on the health-related quality-of-life of patients with primary Sjögren's syndrome. Clin Exp Rheumatol 2005;23(3):351-6.

35. van Nimwegen JF, Arends S, van Zuiden GS, et al. The Impact of Primary Sjögren's Syndrome on Female Sexual Function. Rheumatology (Oxford). 2015;54(7):1286-93.

36. Visser E-S, Visser R, van Lier HJJ, Otten HM. Modern scleral lenses part I: Clinical features. Eye Contact Lens Sci Clin Pract 2007; 33:13-20.

37. Ng A, Sorbara L. Case report: Using scleral lens wear to managing severe ocular dryness secondary to an autoimmune disorder. Cont Lens Anter Eye 2018;41;S1:S50.

38. Wang Y, Kornberg DL, St Clair RM, et al. Corneal nerve structure and function after long-term wear of fluid-filled scleral lens. Cornea 2015;34(4):427-32.

39. Situ P, Simpson TL, Fonn D, et al. Conjunctival and corneal pneumatic sensitivity is associated with signs and symptoms of ocular dryness. Invest Ophthalmol Vis Sci 2008; 49:2971-76.

40. Labbé A, Alalwani H, Van Went C, et al. The relationship between subbasal nerve morphology and corneal sensation in ocular surface disease. Invest Ophthalmol Vis Sci 2012; 53:4926-31.

41. Labbé A, Liang Q, Wang Z, et al. Corneal nerve structure and function in patients with non-Sjogren dry eye: Clinical correlations. Invest Ophthalmol Vis Sci 2013; 54:5144-50.

42. Villani E, Galimberti D, Viola F, et al. The cornea in Sjogren's syndrome: an in vivo confocal study. Invest Ophthalmol Vis Sci 2007; 48:2017-22.

43. Tuisku IS, Konttinen YT, Konttinen LM, et al. Alterations in corneal sensitivity and nerve morphology in patients with primary Sjögren's syndrome. Exp Eye Res 2008; 86:879-85.

44. Ormerod LD, Fong LP, Foster CS. Corneal infection in mucosal scarring disorders and Sjogren's syndrome. Am J Ophthalmol 1988;105(5):512-8.

45. Rosenthal P, Cotter JM, Baum J. Treatment of persistent corneal epithelial defect with extended wear of a fluid-ventilated gas-permeable scleral contact lens. Am J Ophthalmol 2000;130:33-41.
46. Kalwerisky K, Davies B, Mihora L, et al. Demartelaere S. Use of the Boston Ocular Surface Prosthesis in the management of severe periorbital thermal injuries: a case series of 10 patients. Ophthalmology 2012;119:516-21.

47. Zimmerman AB, Marks A. Microbial Keratitis secondary to unintended poor compliance with scleral gas-permeable contact lenses. Eye Contact Lens Sci Clin Pract 2014;40:e1-4.

48. Severinsky B, Behrman S, Frucht-Pery J, Solomon A. Scleral contact lenses for visual rehabilitation after penetrating keratoplasty: long term outcomes. Contact Lens Anterior Eye 2014;37:196-02.

49. Farhat B, Sutphin JE. Deep anterior lamellar keratoplasty for acanthamoeba keratitis complicating the use of Boston scleral lens. Eye Contact Lens 2014;40:e5-7.

50. Fadel D, Toabe M. Compliance using scleral lenses. J Cont Lens Res Sci 2018 April;2(1):e22-29.

51. Fernandes M, Sharma S. Polymicrobial and microsporidial keratitis in a patient using Boston scleral contact lens for Sjogren's syndrome and ocular cicatricial pemphigoid. Contact Lens Anterior Eye 2013;36(2):95-7.

52. Sharma S, Das S, Joseph J, Vemuganti GK, et al. Microsporidial keratitis: need for increased awareness. Survey Ophthalmol 2011;56:1-22.

53. Fadel D. Scleral lens issues and complications related to a non-optimal fitting relationship between the lens and ocular surface. Eye Contact Lens 2019;45(3):152-63.

54. Foulks GN, Forstot SL, Donshik PC, et al. Clinical guidelines for management of dry eye associated with Sjögren Disease. Ocul Surf;2015:13(2):118-32.

55. Choi JH, Kim JH, Li Z, et al. Efficacy of the mineral oil and hyaluronic acid mixture eye drops in murine dry eye. Korean J Ophthalmol 2015;29(2):131-7.

56. Lanzini M, Curcio C, Colabelli-Gisoldi RA, et al. In vivo and impression cytology study on the effect of compatible solutes eye drops on the ocular surface epithelial cell quality in dry-eye patients. Mediators Inflamm 2015;2015:351424.

57. Martin E, Oliver KM, Pearce EI, Tomlinson A, et al. Effect of tear supplements on signs, symptoms and inflammatory markers in dry eye. Cytokine 2018 May;105:37-44.

58. Caffery B, Harthan J, Srinivasan S, et al. sjögren's syndrome in optometric practices in North America. Contact Lens Anterior Eye 2018;41(6):518-26.

59. Benitez-Del-Castillo JM. How to promote and preserve eyelid health. Clin Ophthalmol 2012;6:1689-98.

J Cont Lens Res Sci Vol 4(2):e12-e22; August 25, 2020

This article is distributed under the terms of the Creative Commons Attribution-Non Commercial 4.0 International License.CFadel and Barnett. 
60. Maher TN. The use of tea tree oil in treating blepharitis and meibomian gland dysfunction. Oman J Ophthalmol 2018;11(1):11-15.

61. Romanowski EG, Stella NA Yates KA, et al. In vitro evaluation of a hypochlorous acid hygiene solution on established biofilms. Eye Contact Lens 2018;44 Suppl 2:S187-91.

62. Murphy O, O’Dwyer V, Lloyd-McKernan A. The efficacy of tea tree face wash, 1, 2-Octanediol and microblepharoexfoliation in treating demodex folliculorum blepharitis. Contact Lens Anter Eye 2018;41(1):77-82.

63. Alghamdi YA, Camp A, Feuer W, et al. Compliance and subjective patient responses to eyelid hygiene. Eye Contact Lens 2017;43(4):213-17.

64. Qiu W, Liu Z, Ao M, et al. Punctal plugs versus artificial tears for treating primary Sjögren's syndrome with keratoconjunctivitis sicca: a comparative observation of their effects on visual function. Rheumatol Int 2013;33:2543-8.

65. Marsh P, Pflugfelder SC. Topical Nonpreserved methylprednisolone therapy for keratoconjunctivitis sicca in Sjögren syndrome. Ophthalmology 1999;106:811-6.

66. Hong S, Kim T, Chung SH, et al. Recurrence after topical non-preserved methylprednisolone therapy for keratoconjunctivitis sicca in Sjögren's syndrome. J Ocul Pharmacol Ther 2007;23:78-82.

67. Kunert KS, Tisdale AS, Gipson IK. Goblet cell numbers and epithelial proliferation in the conjunctiva of patients with dry eye syndrome treated with cyclosporine. Arch Ophthalmol 2002;120:330-37.

68. Gündüz K, Ozdemir O. Topical cyclosporin treatment of keratoconjunctivitis sicca in secondary Sjögren's syndrome. Acta Ophthalmol 1994;72:438-42.
69. Sall K, Stevenson OD, Mundorf TK, Reis BL. Two multicenter, randomized studies of the efficacy and safety of cyclosporine ophthalmic emulsion in moderate to severe dry eye disease. CsA Phase 3 Study Group. Ophthalmology 2000;107:631-39.

70. Stevenson D, Tauber J, Reis BL. Efficacy and safety of cyclosporine a ophthalmic emulsion in the treatment of moderate-to-severe dry eye disease: a dose-ranging, randomized trial. The Cyclosporin A Phase 2 Study Group. Ophthalmology 2000;107:967-74.

71. Perez VL, Pflugfelder SC, Zhang S, et al. Lifitegrast, a novel integrin antagonist for treatment of dry eye disease. Ocul Surf 2016;14:207-15.

72. Stern ME, Gao J, Schwalb TA, et al. Conjunctival T-cell populations in Sjögren's and non-Sjögren's patients with dry eye. Invest Ophthalmol Vis Sci 2002;43:2609-14.

73. Tauber J, Karpecki P, Latkany R, et al. Lifitegrast ophthalmic solution $5.0 \%$ versus placebo for treatment of dry eye disease: Results of The Randomized Phase III OPUS-2 Study. Ophthalmology 2015;122:2423-31.

74. Holland EJ, Luchs J, Karpecki PM, Nichols KK, et al. Lifitegrast for the treatment of dry eye disease: Results of a Phase III, Randomized, Double-Masked, Placebo-Controlled Trial (OPUS-3). Ophthalmology 2017 Jan;124(1):53-60.

75. Tsubota K, Goto E, Fujita H, et al. Treatment of dry eye by autologous serum application in Sjögren's syndrome. Br J Ophthalmol 1999;83(4):390-5.

76. Hwang J, Chung SH, Jeon S, Kwok SK, et al. Comparison of clinical efficacies of autologous serum eye drops in patients with primary and secondary Sjögren syndrome. Cornea 2014;33(7):663-7. 